\title{
Brain mechanisms of the Tip-of-the-Tongue state: An electroencephalography-based source localization study
}

\author{
Stanislav A. Kozlovskiy ${ }^{*}$, Sophie D. Shirenova, \\ Anastasia K. Neklyudova, Alexander V. Vartanov \\ Faculty of Psychology, Lomonosov Moscow State University, Moscow, Russia \\ *Corresponding author. E-mail: stas@psy.msu.ru
}

Background. The Tip-Of-the-Tongue (TOT) state occurs when a person fails to retrieve a familiar word, e.g., a name, from long-term memory, while knowing perfectly well that the forgotten word exists in memory and being able to report some information about it (semantic associations, the first letter, the number of syllables, etc.).

Objective and Method. In the present work, we studied the activation of brain structures during the TOT state. The participants $(\mathrm{N}=20$; age $21.5 \pm 4.1)$ viewed portraits of movie stars whose names they were asked to remember. Event related potentials (ERP) were registered in three conditions: 1 ) the participant remembered the name; 2 ) the participant did not know the name; 3) the participant knew the name but could not remember it (TOT-state). The sources of cortical activation were computed (dSPM algorithm).

Results. Time intervals demonstrating significant differences (t-test) in activation among the three conditions were calculated for each activated area, so that up to four different stages of processing could be delineated. According to our analysis, face perception involves activation of the visual cortex (left cuneus and right precuneus cortices), banks of the superior temporal sulci, poles of frontal and temporal lobes, and fusiform gyrus. The early activation does not depend on the successful retrieval of the name. A second increase in activation of the visual cortex is present at a later stage of processing, when name retrieval fails or if it is impeded.

Conclusion. We have shown that successful face recognition involves activation of the posterior cingulate cortex and the isthmus of the cingulate cortex in both hemispheres. Additionally, the parahippocampal gyrus is less active at the early stages and more active at the later stages of processing in the TOT-state, when name retrieval from the long-term memory fails.

Keywords: tip-of-the-tongue (TOT), memory retrieval, verbatim recollection, electroencephalography (EEG), source localization, event related potentials (ERP), posterior cingulate cortex, parahippocampal gyrus, isthmus of cingulate gyrus 


\section{Introduction}

When a person fails to remember a familiar word, a specific subjective state may appear which is known as the Tip-Of-the-Tongue (TOT) phenomenon. In this state, the person is able to retrieve some information about the forgotten word (its first letter, the number of syllables, etc.). S/he can also recall synonyms, which are are immediately rejected as incorrect (Freud, 1904) and correctly report some morphologic characteristics of the word, such as the position of the accented syllable (Brown \& McNeill, 1966). This phenomenon is non-specific to culture and can be observed worldwide. Its frequency of occurrence increases with age: elderly people experience it more often (Salthouse \& Mandell, 2013).

Although this phenomenon has been known for a long time, the first person who shifted the focus of attention to it and popularized such a feature of human memory was the Russian writer Anton Chekhov, who mentioned TOT in his humorous novel "A Horsey Name" (Chekhov, 1885). The main character could not recall a surname, but remembered that it was somehow related to horses. Then he tried to remember every surname that seemed associated with horses; in the end the answer turns out to be "Hayes". In the scientific literature, the first author who described the TOT phenomenon was William James (James, 1890). There are a variety of models in contemporary cognitive science that try to explain this phenomenon. Up to five different theories are currently known, which can be roughly divided into two groups (Schwartz, 1999).

The first three theories can be categorized as "direct-access approaches". They are focused on the problem of memory access. The blocking hypothesis (Jones, 1989) suggests that TOT occurs because a wrong word which sounds very similar comes to mind and blocks the retrieval of the correct word. The incomplete activation theory (Brown \& McNeill, 1966) states that TOT occurs when the activation of the target word's phonological representation is poorer than the activation of words similar in meaning or in pronunciation. Although the target word is not retrieved, a subject can sense its presence in the mind. According to the transmission deficit model (Burke, MacKay, Worthley, \& Wade, 1991), the verbatim recollection is a two-stage process. At the first stage, the semantic level of memory is activated where the core of the concept is recalled. Then the phonological level activates and the meaning of the word is translated into a verbal code. The TOT phenomenon occurs because of the failure of information transition from the semantic to the phonological levels.

The second group of theories emphasizes, instead of access to information in long-term memory interference with it. The cue familiarity hypothesis (Metcalfe, Schwartz, \& Joaquim, 1993) suggests that the request to memory retrieval itself may seem to be very familiar such that a person has the feeling that the information is contained in his/her memory and so can be retrieved easily. According to this hypothesis, the TOT phenomenon emerges because of the difficulties of retrieval. Finally, the accessibility heuristic theory (Koriat, 1993; 1995) suggests that the more different information comes to mind while trying to recall the right word, the stronger the TOT-phenomenon is.

Although there are various experimental data and theoretical considerations in cognitive psychology related to this phenomenon, its brain mechanisms are not fully understood. Present psychophysiological studies mainly describe the charac- 
teristics of event-related potentials (ERPs) or brain rhythms. For example, it was shown that in the condition of a successful recall compared to the situation when a subject did not know the right name, differences are observed in P2, P3, and N450 of ERPs (Bujan, Galdo-Alvarez, Lindin, \& Diaz, 2012). According to other authors (Resnik, Bradbury, Barnes, \& Leff, 2014), positive depression of alpharhythm occurs during the TOT condition, which was interpreted as a manifestation of an ongoing search for semantic information. Psychophysiological data on activation of brain structures are still controversial. For instance, according to Galdo-Alvarez, Lindín, \& Díaz (2011), during the retrieval of a name to match a face the following areas are active: the posterior temporal area, the insula, lateral and medial prefrontal areas, the medial temporal lobe, the anterior cingulate cortex (ACC), and the supplementary motor area (SMA). According to Lindín, Díaz, Capilla, Ortiz, and Maestú (2010), a slightly different list of activated regions was found during this process: left temporal and frontal areas, bilateral parahippocampal gyrus, right fusiform gyrus, bilateral occipital, left temporal, as well as right frontal and parietal areas.

The present study investigated the activation pattern of brain structures during the TOT phenomenon while recollecting a name to match a face. The data were compared to brain activation patterns in control conditions of successful name retrieval and perception of unfamiliar names.

\section{Method}

Twenty volunteers (age 21.5 \pm 4.1; 10 males and 10 females) were recruited for this study. All participants were right-handed. They were healthy, with normal or corrected-to-normal vision acuity, with no history of neurological or psychiatric disorders. All participants gave their informed consent prior to their inclusion in the study, and did not report fatigue due to insufficient sleep. None of the participants were familiar with the protocols used in the study.

Seventy portraits of contemporary British and Hollywood movie stars (35 male and 35 female celebrities) were selected on the basis of normative familiarity judgments obtained by asking independent subjects. The participants viewed the stimuli from a distance of $70 \mathrm{~cm}$. The angular size of the stimuli was 25 angular degrees in height and 16 angular degrees in width. All the stimuli were monochromatic. The background color was black. The actors were photographed face forward. All the pictures were adjusted by eye level and brought to a common format. The faces had no emotional expression (see Figure 1).

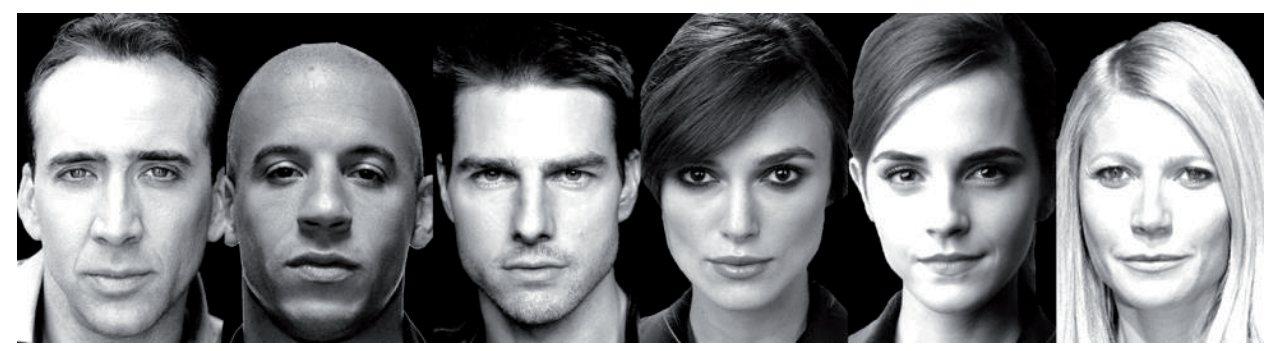

Figure 1. Examples of the actors' faces which were shown to the participants 
The participants were asked to remember the names of the actors. They responded by pressing one of the three buttons:

1. Answer "Know": I remember the name;

2. Answer "DK" [don't know]: I do not know the name;

3. Answer "TOT" [tip-of-the-tongue]: I knew the name, but I don't remember it now.

In order to make the responses automatic the participants were first asked to complete a training session. The task in this session was the same as in the main one. A different set of stimuli was presented for the training session: pictures of famous Russian and Soviet actors (15 male and 15 female faces). The training session lasted 4 minutes. The main session lasted 15 minutes.

EEG registration procedure. Each photograph appeared on the screen for 800 $\mathrm{ms}$, followed by a 2,000 ms pause. For this time period, a fixation point was presented on a black screen. During the pause, the participant gave one of the three possible answers. The stimuli were presented in quasi-random order. Each photograph was shown four times (120 presentations in the training session and 280 presentations in the main session). During the sessions, the EEG registration was executed according to the $10-20 \%$ system using 19 active channels. The stimuli were shown via Presentation ${ }^{\circledast}$ software (version 18.2; Neurobehavioral Systems, Inc.; Berkeley, CA). The event-related potentials (ERPs) were averaged for the three conditions ("DK", "Know", “TOT”) for each participant.

Source localization procedure. The 3D-coordinates of the sources of brain activity were computed for the participants individually for each condition using the dSPM algorithm (Dale et al, 2000). Computed source coordinates were averaged and applied to an averaged brain surface anatomy model (ICBM152) using Brainstorm Software (Tadel, Baillet, Mosher, Pantazis, \& Leahy, 2011; http://neuroimage. usc.edu/brainstorm). For each of the three possible answers, graphs were generated of activation dynamics for the 34 gyral-based regions of interest (ROIs) in each hemisphere. The outlines of the ROIs were determined according to the coordinates of the Desikan-Killiany labeling system (Desikan et al., 2006).

Only the ROIs that were highly active were selected for further analysis (12 brain areas in each hemisphere, see Figure 2): the banks of the superior temporal sulcus, the cuneus cortex, the entorhinal cortex, the frontal pole, the fusiform gyrus, the isthmus of the cingulate cortex, the lingual gyrus, the parahippocampal

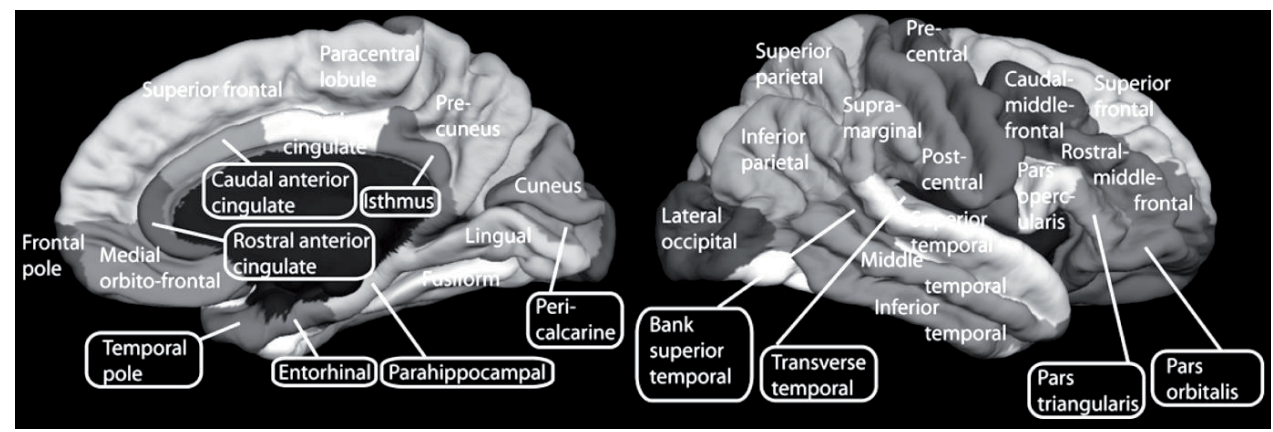

Figure 2. The cortex areas relevant for this study (after graphical materials of Hagmann et al., 2008; with permission of Creative Commons Attribution License) 
gyrus, the pericalcarine cortex, the posterior cingulate cortex, the precuneus cortex, and the temporal pole.

Statistical analysis. For each ROI, time periods of significant differences in activation among three conditions were calculated using Student T-test for independent samples $(\mathrm{p}<0.05 ; \mathrm{N}=20)$.

\section{Results}

No significant differences were found in either hemisphere for the banks of the superior temporal sulci, the poles of frontal and temporal lobes, and the fusiform gyri. T-test also did not reveal any significant differences between the conditions in activation of the left cuneus cortex and the right precuneus cortex (see Figure 3).

It can be observed that at early stages, the brain areas associated with primary visual preprocessing (the cuneus and the precuneus cortices) were active. The fusiform gyrus was active for around $150 \mathrm{~ms}$ after the stimulus. There was high activation of the temporal lobes (the temporal poles and the banks of the superior temporal sulci). Notably, in the left hemisphere the activation of the temporal poles was greater than in the right hemisphere. The activation of the frontal lobes increased during the whole time period that was analyzed.

Table 1. Time periods of significantly differing activation among the conditions (Student T-test). L - left hemisphere; $\mathrm{R}$ - right hemisphere

\begin{tabular}{|c|c|c|c|c|}
\hline Cortex area & $\begin{array}{l}\text { Hemi- } \\
\text { sphere }\end{array}$ & $\begin{array}{l}\text { TOT \& DK } \\
\quad(t, \mathrm{~ms})\end{array}$ & $\begin{array}{l}\text { TOT \& Know } \\
\quad(t, \mathrm{~ms})\end{array}$ & $\begin{array}{l}\text { DK \& Know } \\
\quad(t, \mathrm{~ms})\end{array}$ \\
\hline Cuneus cortex & $\mathrm{R}$ & - & $477-523$ & $501-525,558-568$ \\
\hline $\begin{array}{l}\text { Precuneus } \\
\text { cortex }\end{array}$ & $\mathrm{L}$ & $527-542,688-696$ & $\begin{array}{l}487-568 \\
614-640\end{array}$ & $\begin{array}{l}506-517,552-565 \\
692-698,734-744\end{array}$ \\
\hline \multirow{2}{*}{$\begin{array}{l}\text { Pericalcarine } \\
\text { cortex }\end{array}$} & $\mathrm{L}$ & - & - & $467-480$ \\
\hline & $\mathrm{R}$ & $\begin{array}{c}468-476,645-655 \\
761-767\end{array}$ & - & $464-481$ \\
\hline \multirow{2}{*}{ Lingual gyrus } & $\mathrm{L}$ & $469-478,760-766$ & - & $463-487$ \\
\hline & $\mathrm{R}$ & $464-477,488-499$ & - & $471-480$ \\
\hline \multirow{2}{*}{$\begin{array}{l}\text { Parahippo- } \\
\text { campal gyrus }\end{array}$} & $\mathrm{L}$ & $362-367,458-477$ & $304-313$ & - \\
\hline & $\mathrm{R}$ & $336-363$ & - & - \\
\hline \multirow{2}{*}{$\begin{array}{l}\text { Entorhinal } \\
\text { cortex }\end{array}$} & $\mathrm{L}$ & - & $303-314$ & - \\
\hline & $\mathrm{R}$ & $322-327$ & - & - \\
\hline \multirow{2}{*}{$\begin{array}{l}\text { Isthmus of } \\
\text { the cingulate } \\
\text { cortex }\end{array}$} & $\mathrm{L}$ & $459-465$ & - & $\begin{array}{c}507-519,649-663 \\
693-701\end{array}$ \\
\hline & $\mathrm{R}$ & - & - & $540-549$ \\
\hline \multirow{2}{*}{$\begin{array}{l}\text { Posterior cin- } \\
\text { gulate cortex }\end{array}$} & $\mathrm{L}$ & $452-465$ & - & $649-656$ \\
\hline & $\mathrm{R}$ & $452-462$ & - & $641-658$ \\
\hline
\end{tabular}


Time periods (in ms) during which significant differences among the conditions ("DK", "TOT", "Know") were found are given in Table 1.

Significant differences among the three conditions were found in activation of the pericalcarine cortex, the lingual gyrus, the parahippocampal gyrus, the entorhinal cortex, the isthmus of the cingulate cortex, the posterior cingulate cortex in both hemispheres, and also the right cuneus cortex and the left precuneus cortex (see Figure 4).
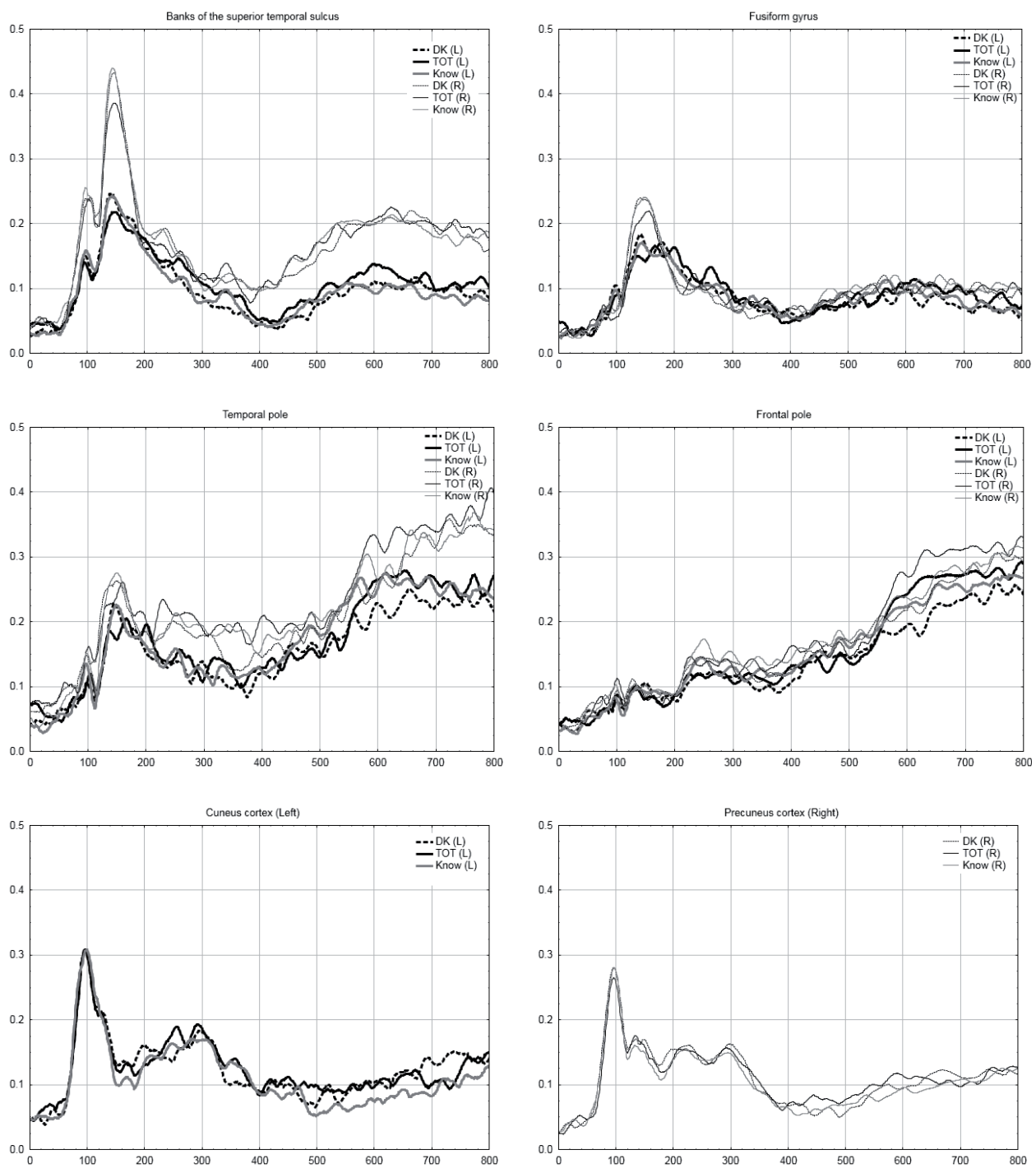

Figure 3. Activation of the brain areas for which no significant differences were found among the conditions. The data was averaged for the participants. The black line - "TOT", the dashed line - "DK", the gray line - "Know". Thick lines - left hemisphere, thin lines right hemisphere 

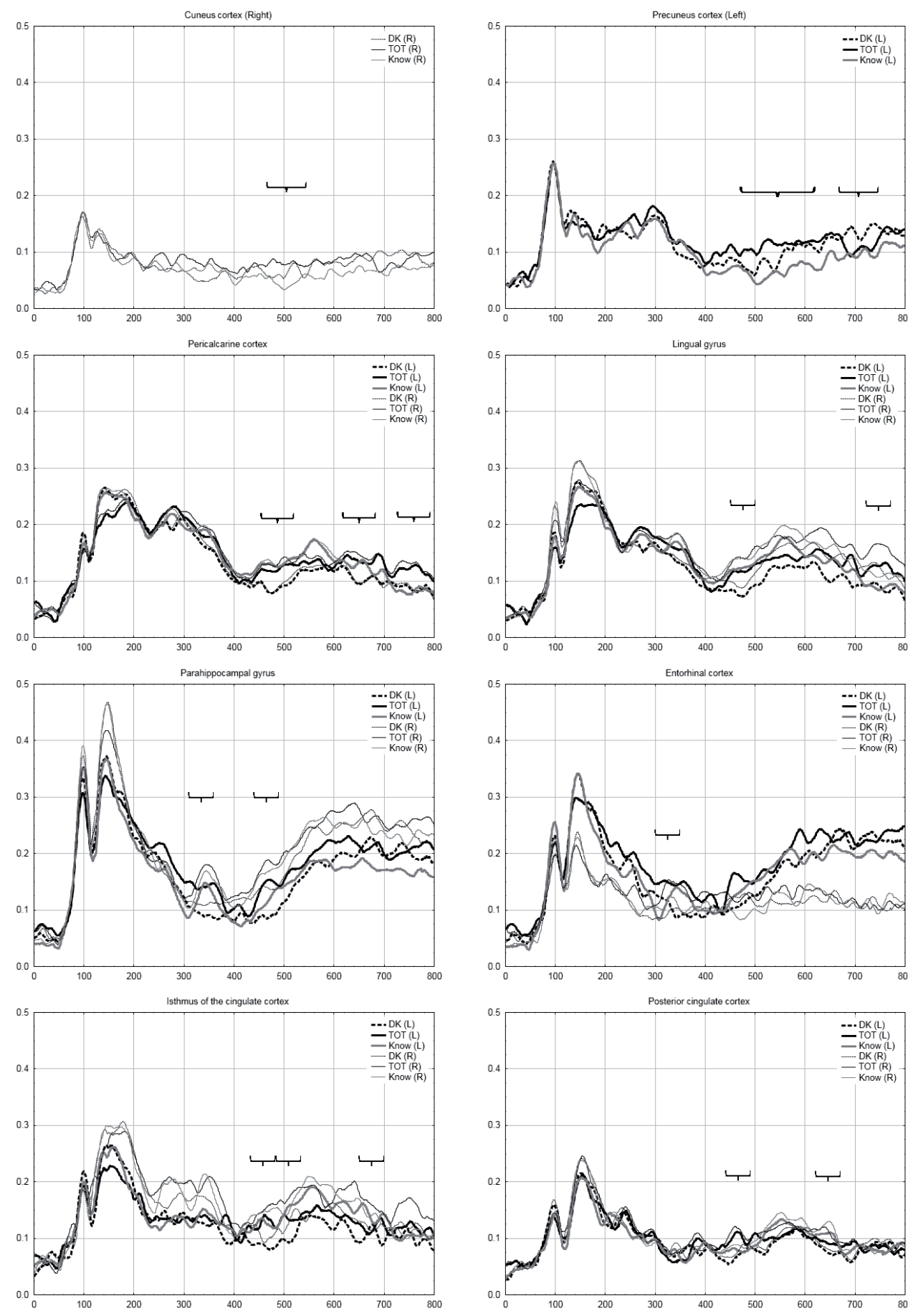

Figure 4. Activation of the brain areas for which significant differences were found among the conditions. The data was averaged for the participants. The black line - "TOT", the dashed line - "DK", the gray line - "Know". Thick lines - left hemisphere, thin lines - right hemisphere. The " $\longleftarrow$ " sign marks time periods of significant differences among the conditions. 


\section{Discussion}

It can be seen that during the analyzed time period ( $800 \mathrm{~ms}$ from the stimulus), activation of the brain structures occurs repeatedly and is relatively synchronized in different brain regions. According to our data, there are four relatively distinct stages when bursts of activation are present: around $100 \mathrm{~ms}$, around 150-200 ms, around $300 \mathrm{~ms}$, and around 400-500 ms. At the first and second stages, no significant differences (t-test) were found in activation of the brain structures among the conditions. The only exception, which does not follow the "four stages" model, is the activation of the frontal poles. It arises almost linearly during the time period analyzed (although it also has faint peaks at the latencies mentioned above). Together with the previously obtained data (Koechlin, 2011), this finding suggests that the activation of the frontal pole should be associated with the fact that the participants had to hold the instruction in their memory and control the task performance. The stages are discussed below.

The first stage is a steep and short increase in activation of the brain structures around $100 \mathrm{~ms}$ from the appearance of the stimulus. This peak is well defined in the cuneus and the precuneus cortices, where it is the highest peak of the analyzed time period. Notably, in the precuneus cortex there were no significant differences between the left and right hemisphere, while the cuneus cortex in the left hemisphere is more active than in the right hemisphere. We suggest that such early activation of the medial occipital and medial inferior parietal cortex at this stage reflects signal detection and its preprocessing. Activation is also present in several other brain regions at this stage (e.g., the lingual gyrus, the parahippocampal gyrus, the entorhinal cortex, the posterior cingulate cortex). However, in these structures this peak is significantly fainter than at the next stages. This may provide evidence that the appearance of the stimulus itself alerts these brain regions, preparing them for the following processing of the stimulus.

The second stage occurs at around 150-200 ms after the stimulus presentation. It is described by a steep increase in activation, which reaches its maximum at 150 $\mathrm{ms}$; then the activation slowly decreases by $200 \mathrm{~ms}$. This is the stage of the maximum activation of the brain. Significant differences ( $t$-test) among the conditions are also absent at this stage. This peak is best defined in the banks of the superior temporal cortex and the parahippocampal gyrus. It is also well defined in the pericalcarine cortex, the lingual gyrus, the fusiform gyrus, the entorhinal cortex, the isthmus of the cingulate cortex, and the posterior cingulate cortex.

Notably, the fusiform gyrus is active only at this stage. During the rest of the analyzed time period, the activation of this brain region does not exceed the noise level. It was shown in multiple studies that the fusiform gyrus is involved in face perception (for a review, see Weiner \& Zilles, 2016). This corresponds with our results, since we used faces as stimuli.

Activation of the banks of the superior temporal sulcus at this stage is higher in the right hemisphere (it is also present in the left hemisphere, but there it is almost half as low in value). While this region has been fully investigated in the left hemisphere (Wernike's area), its function in the right hemisphere requires further analysis. Previous data suggests that this brain region is active, together with the medial 
orbitofrontal cortex, during a face attractiveness assessment task (O'Doherty, Winston, Critchley, Perrett, Burt, \& Dolan, 2003; Kranz \& Ishai, 2006). We did not observe such a coordinated activation in our experiment. A further hypothesis (Karnakh, 2001) can explain the results. It states that the right superior temporal sulcus is involved in the interfacing of the dorsal and ventral streams of visual processing. This suggestion was based on a study of clinical cases of visual spatial neglect. Accordingly, activation of the banks of the superior temporal sulcus in our experiment could be explained as a manifestation of visual stimulus processing. A recent elaboration of this view relates the region's activity to the hippocampal formation (Velichkovsky, Krotkova, Sharaev, \& Ushakov, 2017). Indeed, the parahippocampal gyrus is highly active at this stage, more so than any other analyzed brain region during the whole experiment.

In the well-known study of Quiroga et al. (Quiroga, Reddy, Kreiman, Koch, \& Fried, 2005), where recording electrodes were implanted into the hippocampus, the parahippocampal gyrus, and the entorhinal cortex, it was found that neurons in this area are selectively activated by the faces of specific people. We used photos of famous actors, so the participants had viewed them multiple times regardless of whether they knew their names. Thus, in our experiment the activation of the parahippocampal gyrus may be caused by perception of a familiar face and activation of the information about this actor in long-term memory. Notably, the activation of the parahippocampal gyrus in the TOT state in both hemispheres at this stage was lower than in both other conditions. This finding can testify to an incomplete activation of the semantic network while perceiving a familiar face, with resulting inability to retrieve a name. This interpretation conforms to "the incomplete activation theory" (Brown \& McNeill, 1966), according to which the TOT phenomenon is caused by a low activation of memory traces. However, at the later stages, the activation of this brain region is higher for the TOT condition, which contradicts this theory.

At the third stage a local maximum appears at around $300 \mathrm{~ms}$ from the stimulus, which slowly decreases by $400 \mathrm{~ms}$. This peak is lower in amplitude than the first and second peaks described above. It was observed in activation of the visual cortex structures (the left cuneus cortex, the precuneus cortex, the pericalcarine cortex, and the lingual gyrus). Moreover, in the "DK" condition, this activation is the lowest, and in the "TOT" condition the activation is the highest. This secondary activation of the occipital region could be explained as visual cortex re-entrance (Ivanitskiy, 1976; Edelman, 1989). Apparently, subjects at this stage examine a face with more focal attention, making sure that they have perceived it correctly. This peak is also well expressed in the parahippocampal gyrus and the entorhinal cortex, although in this case, the activation significantly differs ( $\mathrm{t}$-test), depending on whether the name retrieval was successful or not. In the "DK" condition, when a participant decided that s/he doesn't know the target name, this peak is not present in either of these two structures. On the contrary, in the other two conditions this peak of activation was clearly observed. One possible explanation is that at the previous stage a participant who saw and recognized a familiar face, decided that s/ he does not know the target name, and that there is no need to keep searching for 
it in long-term memory. The activation observed in the "Know" and in the "TOT" conditions may reflect a search for a name of a recognized person.

The fourth stage is the last burst of activation of the brain regions during the analyzed time period, and the one of the longest duration. The increase in activations begins around $400 \mathrm{~ms}$ from the stimulus, reaches its maximum by $600 \mathrm{~ms}$ from the stimulus, and slowly decreases by $800 \mathrm{~ms}$. It consists of a 6-to- 8 range of faint peaks of activation. At this stage a secondary activation of the visual cortex was observed (in the lingual gyrus, the pericalcarine cortex, the precuneus cortex, and faintly in the cuneus cortex). Notably, the highest activation was observed in the TOT condition, when a participant failed to retrieve the target name. Apparently, in this case a participant failing to remember the name looks closely at the face again hoping to find a clue to the name in the face of the actor. This hypothesis explains the known fact of alpha-rhythm depression that occurs during the TOT state (Resnik, Bradbury, Barnes, \& Leff, 2014). The parahippocampal gyrus, which was discussed above, is also active, whereby its activation is higher in the TOT condition. This may be explained as an additional attempt to remember the target name. Another explanation of this finding is provided by the accessibility heuristic theory (Koriat, 1993; 1995), described above.

In all three conditions, high activation of the temporal poles was observed, especially in the right hemisphere. According to a meta-analysis (Olson, Plotzker, \& Ezzyat, 2007), this brain region is involved in face perception, but it is mostly associated with socio-emotional processing. In our experiment, activation of the temporal poles may reflect an emotional attitude towards the actor.

At this last stage, the activation of the isthmus of the cingulate cortex and the posterior cingulate cortex was observed, with significant differences among the three conditions. The lowest activation was observed in the "DK" condition and the highest in the "Know" condition. Apparently these brain regions detect whether the retrieval from the long-term memory was successful. This explanation corresponds with data of a previous study (Kozlovskiy, Vartanov, Nikonova, Pyasik, \& Velichkovsky, 2012, 2013) demonstrating that the volume of these brain areas negatively correlates with the number of memory errors. It has also been shown that there are strong causal interactions between the posterior cingulate and the hippocampal formation (Ushakov et al., 2016).

\section{Conclusion}

Retrieving a name by a photograph of a person is a complex task, which consists of multiple interacting cognitive and affective processes, such as visual recognition of a stimulus as a face, assessment of its attractiveness and its emotional expression, recognition of the face, name search in the verbal memory, decision making, and cognitive control. According to our data, this task activates multiple brain regions associated with various brain functional systems: the banks of the superior temporal sulcus, the cuneus cortex, the entorhinal cortex, the frontal pole, the fusiform gyrus, the isthmus of the cingulate cortex, the lingual gyrus, the parahippocampal gyrus, the pericalcarine cortex, the posterior cingulate cortex, the precuneus cortex, and the temporal pole. 
Face perception involves activation of the visual cortex (the left cuneus and the right precuneus cortices), the banks of the superior temporal sulci, the poles of frontal and temporal lobes, and the fusiform gyri, which does not depend on whether the retrieval of the name from long-term memory was successful. If name retrieval fails, a second increase in activation of the visual cortex is present at later time intervals (right cuneus cortex). We have shown that successful face recognition involves activation of the posterior cingulate cortex and isthmus of the cingulate cortex in both hemispheres. According to our results, the parahippocampal gyrus is less active at the early stages and more active at the later stages of processing, when name retrieval from long-term memory fails.

Unfortunately, the method that was used does not allow the analysis of processing within the subcortical structures. For instance, we were not able to analyze the activation of the hippocampus, the brain structure that is massively involved in memory task solution. Therefore, we plan to overcome these limitations by completing our experimental framework with data of a functional MRI study and an EEG study with a large number of active channels.

\section{Acknowledgments}

This study was partially supported by the Russian Science Foundation (project RScF16-18-00066). We wish to express our gratitude for the criticism and enriching discussion of our results by B.M. Velichkovsky.

\section{References}

Brown, R., \& McNeil, D. (1966). The "tip of the tongue" phenomenon. Journal of Verbal Learning and Verbal Behavior, 5(4), 325-337. doi: 10.1016/S0022-5371(66)80040-3

Bujan, A., Galdo-Alvarez, S., Lindin, M., \& Diaz, F. (2012). An event-related potentials study of face naming: Evidence of phonological retrieval deficit in the tip-of-the-tongue state. Psychophysiology, 49(7), 980-990. doi: 10.1111/j.1469-8986.2012.01374.x

Burke, D.M., MacKay, D.G., Worthley, J. S., \& Wade, E. (1991). On the tip of the tongue: What causes word finding failures in young and older adults? Journal of memory and language, 30(5), 542-579. doi: 10.1016/0749-596X(91)90026-G

Chekhov, A.P. (1915). A horsey name. In M. Fell (Ed.), Russian silhouettes; more stories of Russian life. New York: C. Scribner's sons. (Original work published 1885)

Dale, A.M., Liu A.K., Fischl, B.R., Buckner, R.L., Belliveau, J.W., Lewine, J.D., \& Halgren, E. (2000). Dynamic statistical parametric mapping: Combining fMRI and MEG for highresolution imaging of cortical activity. Neuron, 26(1), 55-67. doi: 10.1016/S0896-6273(00)81138-1

Desikan, R.S., Ségonne, F., Fischl, B., Quinn, B.T., Dickerson, B.C., Blacker, D. ... Killiany, R.J., (2006). An automated labeling system for subdividing the human cerebral cortex on MRI scans into gyral based regions of interest. Neuroimage, 31(3), 968-980. doi: 10.1016/j. neuroimage.2006.01.021

Edelman, G.M. (1989). The remembered present. A biological theory of consciousness. New York: Basic Books.

Freud, S. (1904). Zur Psychopathologie des Alltagslebens [On psychopathology of everyday life]. Berlin: S. Karger. 
Galdo-Alvarez, S., Lindín M., \& Díaz F. (2011). Brain dynamics associated with face-naming and the tip-of-the-tongue state. Psicothema, 23(2), 189-195.

Hagmann, P., Cammoun, L., Gigandet, X., Meuli, R., Honey, C.J., Wedeen, V.J., \& Sporns, O. (2008). Mapping the structural core of human cerebral cortex. PLOS Biology, 6(7), 14791493. doi: 10.1371/journal.pbio.0060159

Karnath, H.-O. (2001). New insights into the functions of the superior temporal cortex. Nature Reviews Neuroscience, 2(8), 568-576. doi: 10.1038/35086057

Kranz, F., \& Ishai, A. (2006). Face perception is modulated by sexual preference. Current Biology, 16(1), 63-68. doi: 10.1016/j.cub.2005.10.070

Koechlin, E. (2011). Frontal pole function: What is specifically human? Trends in Cognitive Sciences, 15(6), 241. doi: 10.1016/j.tics.2011.04.005

Ivanitskiy, A.M. (1976). Mozgovye mekhanizmy otsenki signalov [Brain mechanisms of signal evaluation]. Moscow: Meditsina.

James, W. (1890). The principles of psychology, New York: H. Holt and Company. doi: 10.1037/11059-000

Jones, G.V. (1989). Back to Woodworth: Role of interlopers in the tip-of-the-tongue phenomenon. Memory \& Cognition, 17(1), 69-76. doi: 10.3758/BF03199558

Koriat, A. (1993). How do we know that we know? The accessibility model of the feeling of knowing. Psychological Review, 100, 609-639. doi: 10.1037/0033-295X.100.4.609

Koriat, A. (1995). Dissociating knowing and the feeling of knowing: Further evidence for the accessibility model. Journal of Experimental Psychology: General, 124, 311-333. doi: 10.1037/0096-3445.124.3.311

Kozlovskiy S.A., Vartanov A.V., Nikonova E.Y., Pyasik M.M., \& Velichkovsky B.M. (2012). The cingulate cortex and human memory processes. Psychology in Russia: State of the Art, 5, 231-243. doi: 10.11621/pir.2012.0014

Kozlovskiy S.A., Vartanov A.V., Nikonova E.Y., Pyasik M.M., \& Velichkovsky B.M. (2013). Anatomical characteristics of cingulate cortex and neuropsychological memory tests performance. Procedia - Social and Behavioral Sciences, 86(10), 128-133. doi: 10.1016/j. sbspro.2013.08.537

Lindín, M., Díaz, F., Capilla, A., Ortiz, T., \& Maestú, F., (2010). On the characterization of the spatio-temporal profiles of brain activity associated with face naming and the tip-of-thetongue state: A magnetoencephalographic (MEG) study. Neuropsychologia, 48(6), 17571766. doi: 10.1016/j.neuropsychologia.2010.02.025

Metcalfe, J., Schwartz, B.L., \& Joaquim, S.G. (1993). The cue familiarity heuristic in metacognition. Journal of Experimental Psychology: Learning, Memory, \& Cognition, 19, 851-861. doi: 10.1037//0278-7393.19.4.851

O’Doherty, J., Winston, J., Critchley, H., Perrett, D., Burt, D.M., \& Dolan, R.J. (2003). Beauty in a smile: The role of medial orbitofrontal cortex in facial attractiveness. Neuropsychologia, 41(2), 147-155. doi: 10.1016/S0028-3932(02)00145-8

Olson, I.R., Plotzker, A., \& Ezzyat, Y. (2007). The enigmatic temporal pole: A review of findings on social and emotional processing. Brain, 130(7), 1718-1731. doi: 10.1093/brain/awm052

Resnik, K., Bradbury, D., Barnes, G.R., \& Leff, A.P. (2014). Between thought and expression, a magnetoencephalography study of the "tip-of-the-tongue" phenomenon. Journal of Cognitive Neuroscience, 26(10), 2210-2223. doi: 10.1162/jocn_a_00611

Quiroga, R.Q., Reddy, L., Kreiman, G., Koch, C., \& Fried, I. (2005). Invariant visual representation by single neurons in the human brain. Nature, 435(7045), 1102-1107. doi: 10.1038/ nature 03687 
Salthouse, T.A., \& Mandell A.R. (2013). Do age-related increases in tip-of-the-tongue experiences signify episodic memory impairments? Psychological Science, 24(12), 2489-2497. doi: $10.1177 / 0956797613495881$

Schwartz, B.L. (1999). Sparkling at the end of the tongue: The etiology of tip-of-the-tongue phenomenology. Psychonomic Bulletin \& Review, 6(3), 379-393. doi: 10.3758/BF03196771

Sugiura, M., Shah, N.J., Zilles, K., \& Fink, G.R. (2005). Cortical representations of personally familiar objects and places: Functional organization of the human posterior cingulate cortex. Journal of Cognitive Neuroscience, 17(2), 183-198. doi: 10.1162/0898929053124956

Tadel F., Baillet S., Mosher J.C., Pantazis D., \& Leahy R.M. (2011). BrainStorm: a user-friendly application for MEG/EEG analysis. Computational Intelligence and Neuroscience, 2011, 1-13. doi: $10.1155 / 2011 / 879716$

Ushakov, V.L., Sharaev, M. G., Kartashov, S.I., Zavyalova, V.V., Verkhlyutov, V.M., \& Velichkovsky, B.M. (2016) Dynamic causal modeling of hippocampal links within the human default mode network: Lateralization and computational stability of effective connections. Frontiers in Human Neuroscience, 10, 1-14. doi: 10.3389/fnhum.2016.00528

Velichkovsky, B.M., Krotkova, O.A., Sharaev, M.G., \& Ushakov, V.L. (2017). In search of the "I": Neuropsychology of lateralized thinking meets Dynamic Causal Modeling. Psychology in Russia: State of the Art. This issue.

Weiner, K.S., \& Zilles, K. (2016). The anatomical and functional specialization of the fusiform gyrus. Neuropsychologia, 83(3), 48-62. doi: 10.1016/j.neuropsychologia.2015.06.033

Original manuscript received December 06, 2016 Revised manuscript accepted February 18, 2017

First published online September 30, 2017 